\title{
Piezoresistance in p-type silicon revisited
}

Richter, Jacob; Pedersen, Jesper; Brandbyge, Mads; Thomsen, Erik Vilain; Hansen, Ole

Published in:

Journal of Applied Physics

Link to article, DOI:

$10.1063 / 1.2960335$

Publication date:

2008

Document Version

Publisher's PDF, also known as Version of record

Link back to DTU Orbit

Citation (APA):

Richter, J., Pedersen, J., Brandbyge, M., Thomsen, E. V., \& Hansen, O. (2008). Piezoresistance in p-type silicon revisited. Journal of Applied Physics, 104(2), 023715. https://doi.org/10.1063/1.2960335

\section{General rights}

Copyright and moral rights for the publications made accessible in the public portal are retained by the authors and/or other copyright owners and it is a condition of accessing publications that users recognise and abide by the legal requirements associated with these rights.

- Users may download and print one copy of any publication from the public portal for the purpose of private study or research.

- You may not further distribute the material or use it for any profit-making activity or commercial gain

- You may freely distribute the URL identifying the publication in the public portal

If you believe that this document breaches copyright please contact us providing details, and we will remove access to the work immediately and investigate your claim. 


\title{
Piezoresistance in p-type silicon revisited
}

\author{
J. Richter, ${ }^{1, a)}$ J. Pedersen, ${ }^{1}$ M. Brandbyge,${ }^{1}$ E. V. Thomsen, ${ }^{1}$ and O. Hansen ${ }^{1,2}$ \\ ${ }^{1}$ Department of Micro- and Nanotechnology, DTU Nanotech Building 345 East, Technical University of \\ Denmark, DK-2800 Kgs. Lyngby, Denmark \\ ${ }^{2}$ Danish National Research Foundation's Center for Individual Nanoparticle Functionality (CINF), \\ Technical University of Denmark, DK-2800 Kgs. Lyngby, Denmark
}

(Received 28 January 2008; accepted 28 May 2008; published online 30 July 2008)

\begin{abstract}
We calculate the shear piezocoefficient $\pi_{44}$ in $p$-type Si with a $6 \times 6 \mathbf{k} \cdot \mathbf{p}$ Hamiltonian model using the Boltzmann transport equation in the relaxation-time approximation. Furthermore, we fabricate and characterize $p$-type silicon piezoresistors embedded in a (001) silicon substrate. We find that the relaxation-time model needs to include all scattering mechanisms in order to obtain correct temperature and acceptor density dependencies. The $\mathbf{k} \cdot \mathbf{p}$ results are compared to results obtained using a recent tight-binding (TB) model. The magnitude of the $\pi_{44}$ piezocoefficient obtained from the TB model is a factor of 4 lower than experimental values; however, the temperature and acceptor density dependencies of the normalized values agree with experiments. The $6 \times 6$ Hamiltonian model shows good agreement between the absolute value of $\pi_{44}$ and the temperature and acceptor density dependencies when compared to experiments. Finally, we present a fitting function of temperature and acceptor density to the $6 \times 6$ model that can be used to predict the piezoresistance effect in p-type silicon. (C) 2008 American Institute of Physics. [DOI: 10.1063/1.2960335]
\end{abstract}

\section{INTRODUCTION}

Piezoresistance in silicon and germanium was discovered by Smith in $1954 .^{1}$ Since then there has been academic and industrial interest in the characterization and exploitation of piezoresistance in silicon and other semiconductor materials. The interest from industry is due to the large piezoresistive response of silicon that favors a large sensitivity of microelectromechanical system (MEMS) sensors. ${ }^{2}$ The academic focus is due to scarcity of reliable experimental results and disagreement between measurements and theoretical models, especially in $p$-type silicon. Yet another important issue is that the results from the theoretical models need to be available in a form that is directly usable for experimentalists. By increasing the complexity of materials as technology matures, the need for a reliable theoretical piezoresistive model becomes important. For example, experimental data on strained silicon crystals ${ }^{3}$ and silicon nanowires ${ }^{4}$ show a significant increase in the piezoresistive effect. In order to understand the effect in the new material structures that nanotechnology provides, a fully developed physical model for bulk material, in particular $p$-type silicon, is needed.

In $p$-type silicon the piezoresistive effect is mainly described by the shear piezocoefficient $\pi_{44}$. The shear piezocoefficient is known to be dependent on temperature and doping level. ${ }^{5}$ Thus a model is needed in order to predict MEMS device sensitivity. For prediction of the sensitivity, a model from Kanda ${ }^{6}$ is most commonly used. Kanda ${ }^{6}$ determined a correction factor $P(T, N)$ to describe the effect of temperature $T$ and doping level $N$ on the piezocoefficients. Kanda's ${ }^{6}$ analysis was based on repopulation effects due to stress induced shifts of rigid parabolic bands or valleys, assuming a power law energy dependency of the relaxation time. This

a)Electronic mail:jar@mic.dtu.dk. many-valley model ${ }^{7}$ has proven very successful in describing piezoresistivity in $n$-type silicon, where even the predicted relative magnitudes of the piezocoefficients agree well with experiments. In $p$-type silicon, however, the model is not as successful since the piezoresistivity is due to warpage of the energy surfaces while repopulation effects are unimportant, as already pointed out by Adams. ${ }^{8}$ In spite of this fact the correction factor $P(T, N)$ is often used and quoted in most textbooks on the subject. It is, however, an experimental fact that it severely underestimates the piezocoefficient $\pi_{44}$ at high doping levels; this is very important since optimization of piezoresistive sensors for low $1 / f$ noise favor the use of high doping levels. ${ }^{9}$ Recently, Kozlovskiy et al. ${ }^{10}$ carried out a detailed analytical study of piezoresistance in $p$-type silicon using analytical valence band models of varying complexity, derived from Pikus and Bir, ${ }^{11}$ combined with a power law model for momentum relaxation time, as was also used in previous works. ${ }^{12-14}$ Approximations to the valence band structure valid close to the top of the valence band were used in Refs. 13 and 14, while Toriyama and Sugiyama ${ }^{12}$ used an approximation valid at larger hole energies.

In this paper we calculate the piezocoefficient $\pi_{44}$ in silicon based on a $6 \times 6 \mathbf{k} \cdot \mathbf{p}(6 \times 6)$ model as well as a state-of-the-art tight-binding (TB) model, and fabricate and characterize silicon samples in order to compare theory with experimental results. We calculate the piezoresistance using the Boltzmann transport equation considering different models for the energy-dependent relaxation time. We find that it is vital to include all scattering mechanisms properly in the relaxation time, as also noted by Ohmura. ${ }^{15}$ The results from the two models are compared to experimental data obtained on microfabricated boron doped silicon piezoresistors embedded in a silicon substrate, from own experiments and from Tufte and Stelzer. ${ }^{5}$ The results from the $6 \times 6$ model are used to obtain a fitting function of the piezocoefficient $\pi_{44}$ 
dependency on the temperature and the acceptor density. This simple fit can easily be included in commercial software to predict the output of a designed piezoresistive MEMS component.

\section{THEORY}

\section{A. Piezoresistivity}

By applying a tensorial strain $\epsilon_{i j}$ or stress $X_{i j}$ to a silicon crystal, the resistivity and conductivity tensors change. These changes are in the low stress linear regime characterized by the fourth order piezoresistance tensor $\pi_{i j k l}$. The resistivity $\varrho_{i j}$ and conductivity $\sigma_{i j}$ tensors are

$$
\begin{aligned}
& \varrho_{i j}=\varrho_{i j}^{(0)}+\Delta \varrho_{i j}=\varrho_{0}\left(\delta_{i j}+\pi_{i j k l} X_{k l}\right), \\
& \sigma_{i j}=\sigma_{i j}^{(0)}+\Delta \sigma_{i j}=\sigma_{0}\left(\delta_{i j}-\pi_{i j k l} X_{k l}\right),
\end{aligned}
$$

where $i, j, k, l \in\{1,2,3\}, \delta_{i j}$ is Kronecker's delta, and $\Delta \varrho_{i j}$ and $\Delta \sigma_{i j}$ are the stress induced resistivity and conductivity changes, respectively. The superscript (0) indicates unstrained condition, and $\sigma_{0}$ and $\varrho_{0}$ are the scalar conductivity and resistivity, respectively. By using six-vector notation and by applying the symmetry of the silicon crystal, ${ }^{16}$ the piezoresistance tensor is simplified to a $6 \times 6$ matrix $\pi_{\eta \mu}$ with only three independent coefficients $-\pi_{11}, \pi_{12}$, and $\pi_{44}$. We determine the shear piezocoefficient using

$$
\pi_{44}=-\frac{1}{X_{6}} \frac{\Delta \sigma_{6}}{\sigma_{0}}=-\frac{S_{44}}{\epsilon_{6}} \frac{\Delta \sigma_{6}}{\sigma_{0}}=-\frac{S_{44}}{\epsilon_{6}} \frac{\sigma_{6}}{\sigma_{0}},
$$

where the linear relation between stress and strain is used, i.e., $\epsilon_{\eta}=S_{\eta \mu} X_{\mu}$, where $\eta, \mu \in\{1,2,3,4,5,6\}$, and the compliance tensor $S_{\eta \mu}$ has the same structure as the piezoresistance tensor $\pi_{\eta \mu}$. Notice that the six-vector to tensor element relations are $\Delta \sigma_{6}=\Delta \sigma_{x y}, X_{6}=X_{x y}$, and $\epsilon_{6}=2 \epsilon_{x y}{ }^{16}$ From Eq. (2) the shear piezocoefficient $\pi_{44}$ may be calculated from the calculated shear conductance values $\sigma_{6}$ at given values of the shear strain $\epsilon_{x y}$.

\section{B. Conductivity}

Given the dispersion relation $\xi_{n}(\mathbf{k})$, where $\xi_{n}$ is the hole energy, $\mathbf{k}$ is the wavevector, and $n$ refers to the band index, the electrical conductivity tensor may be calculated using the Boltzmann transport equation in the relaxation-time approximation, ${ }^{17}$

$$
\sigma_{i j}=-\frac{e^{2}}{4 \pi^{3} \hbar^{2}} \sum_{n=1}^{3} \int \tau_{\mathbf{m}}(\mathbf{k}, T) \frac{\partial \xi_{n}(\mathbf{k})}{\partial k_{i}} \frac{\partial \xi_{n}(\mathbf{k})}{\partial k_{j}} \frac{\partial f_{0}}{\partial \xi} \mathrm{d} \mathbf{k},
$$

where the sum extends over the three valence bands, and $f_{0}$ is the equilibrium distribution function for holes. The relaxation time $\tau_{\mathbf{m}}$ is most commonly modeled via a simple power law,

$$
\tau_{\mathbf{m}} \propto\left(\frac{\xi_{n}(\mathbf{k})-\xi_{n}^{(0)}}{k_{B} T_{0}}\right)^{s},
$$

where $\xi_{n}^{(0)}$ is the band minimum, $T_{0}=300 \mathrm{~K}$, and the parameter $s \in\{-1 / 2,0,3 / 2\}$. The $s=-1 / 2$ model corresponds to scattering dominated by acoustic phonons and is the model employed by Kanda, ${ }^{6}$ the $s=0$ model assumes a constant relaxation time, and $s=3 / 2$ corresponds to scattering dominated by ionized impurities. Such a crude model is insufficient for several reasons. First, it is a very poor model for nonpolar optical phonon scattering, which is important in silicon near room temperature. Second, the different scattering mechanisms emphasize transport in regions of the band structure rendered unimportant by other scattering mechanisms, and thus the final real transport properties cannot be deduced from individual conductivities evaluated using this model. That is, Matthiessen's rule simply cannot be applied to the integrated quantities. We consequently employ a more detailed model of the relaxation time. The important scattering mechanisms near room temperature are nonpolar optical phonon scattering, acoustical phonon scattering, and ionized impurity scattering. The microscopic scattering rates add; thus the momentum relaxation time is taken as

$$
\frac{1}{\tau_{\mathbf{m}}(\mathbf{k}, T)}=\frac{1}{\tau_{\mathrm{ap}}(\mathbf{k}, T)}+\frac{1}{\tau_{\mathrm{op}}(\mathbf{k}, T)}+\frac{1}{\tau_{I}(\mathbf{k}, T)},
$$

where $1 / \tau_{\mathrm{ap}}, 1 / \tau_{\mathrm{op}}$, and $1 / \tau_{I}$ are the scattering rates due to acoustic phonons, nonpolar optical phonons, and ionized impurities, respectively.

The acoustic phonon scattering rate is ${ }^{18}$

$$
\frac{1}{\tau_{\text {ap }}}=\frac{2 \pi D_{\mathrm{a}}^{2} k_{B} T}{\hbar \rho v_{s}^{2}} g\left(\xi_{\mathbf{k}}\right)
$$

where $D_{a}$ is the acoustic deformation potential, $\rho$ is the mass density, $v_{s}$ is the speed of sound, and $g\left(\xi_{\mathbf{k}}\right)$ is the density of final states. The high density of states in the heavy hole band makes scattering to this band dominant. Thus, for simplicity we take for all three bands the acoustic scattering rate

$$
\frac{1}{\tau_{\mathrm{ap}}(\mathbf{k}, T)}=\frac{1}{\tau_{\mathrm{ap} 0}} \frac{T}{T_{0}} \sqrt{\frac{\xi(\mathbf{k})-\xi_{h h}^{(0)}}{k_{B} T_{0}}},
$$

with $\tau_{\text {ap } 0}=5.6 \times 10^{-13} \mathrm{~s}$ adjusted to reproduce the rates calculated by Hinckley and Singh. ${ }^{19}$ Here $\xi_{h h}^{(0)}$ is the minimum of the heavy hole band. For simplicity we have assumed an overall parabolic band structure for the target heavy hole band, and use the effective mass of the unstrained heavy hole band in the calculation of the density of states of the target band.

The nonpolar optical phonon scattering rate is ${ }^{18}$

$$
\frac{1}{\tau_{\mathrm{op}}}=\frac{\pi D_{o}^{2}}{\rho \omega_{0}}\left[N_{q} g\left(\xi_{\mathbf{k}}+\hbar \omega_{0}\right)+\left(N_{q}+1\right) g\left(\xi_{\mathbf{k}}-\hbar \omega_{0}\right)\right]
$$

where the first term is due to absorption and the second term is due to emission of an optical phonon with the energy $\hbar \omega_{0}$. $D_{o}$ is the optical deformation potential and $N_{q}$ $=1 /\left(\exp \hbar \omega_{0} / k_{B} T-1\right)$ is the phonon occupation probability. Again, scattering to the heavy hole band is dominant; thus for all three bands we obtain a simplified nonpolar optical scattering rate as 


$$
\begin{aligned}
\frac{1}{\tau_{\mathrm{op}}(\mathbf{k}, T)}= & \frac{1}{\tau_{\mathrm{op} 0}}\left[N_{q} \sqrt{\frac{\xi(\mathbf{k})-\xi_{h h}^{(0)}+\hbar \omega_{0}}{k_{B} T_{0}}}\right. \\
& \left.+\left(N_{q}+1\right) \operatorname{Re}\left(\sqrt{\frac{\xi(\mathbf{k})-\xi_{h h}^{(0)}-\hbar \omega_{0}}{k_{B} T_{0}}}\right)\right],
\end{aligned}
$$

with $\tau_{\mathrm{op} 0}=10^{-13} \mathrm{~s}$, which reproduces the rates calculated by Hinckley and Singh. ${ }^{19}$

By assuming full ionization, the Brooks-Herring screened Coulomb potential scattering rate is ${ }^{18}$

$$
\frac{1}{\tau_{I}}=\frac{\pi N_{A} e^{4}}{4 \varepsilon^{2} \hbar k^{4}} g\left(\xi_{\mathbf{k}}\right) L\left(2 \lambda_{D} k\right),
$$

where $\lambda_{D}$ is the Debye length, $\varepsilon$ is the permittivity, and $N_{A}$ is the acceptor density, and where the function $L(x)=\ln (1$ $\left.+x^{2}\right)-x^{2} /\left(1+x^{2}\right)$ is slowly varying for $x>1$. Again, scattering to the heavy hole band is dominant; thus, as an approximate model for all three bands, we write

$$
\frac{1}{\tau_{I}}=\frac{1}{\tau_{I 0}} \frac{k_{0}^{4}}{k^{4}} \frac{N_{A}}{N_{0}} \sqrt{\frac{\xi(\mathbf{k})-\xi_{h h}^{(0)}}{k_{B} T_{0}}} \times L\left(2 \lambda_{D} k\right),
$$

with the parameters $N_{0}=10^{17} \mathrm{~cm}^{-3}, k_{0}=1.0 \AA^{-1}$, and $\tau_{I 0}$ $=1.8 \times 10^{-7} \mathrm{~s}$ calculated using the density of states mass for the heavy hole band. The Debye length is the electrostatic screening length obtained in a linearization of Poisson's equation for the semiconductor. Thus

$$
\frac{1}{\lambda_{D}^{2}} \equiv \frac{e^{2}}{\varepsilon k_{B} T}\left|\frac{\partial h}{\partial \xi_{F}}\right| \simeq \frac{e^{2} N_{A}}{\varepsilon k_{B} T} \frac{\mathcal{F}_{-1 / 2}\left(\xi_{F}\right)}{\mathcal{F}_{1 / 2}\left(\xi_{F}\right)},
$$

where $h$ is the hole density, $\xi_{F}$ is the Fermi level in units of $k_{B} T$, and $\mathcal{F}_{n}(x)$ is the Fermi-Dirac integral of order $n$. In the approximate expression, an overall parabolic band approximation is assumed.

\section{Band structures}

We have employed two different methods to calculate the band structure of silicon-a $6 \times 6 \mathbf{k} \cdot \mathbf{p}$ Hamiltonian model and a recent TB model. The main reason for the comparison is that TB is used extensively to model transport in $\mathrm{Si}$ nanostructures and it is thus interesting to access the performance of TB.

For the $6 \times 6$ model we closely follow Hinckley and Singh ${ }^{19}$ where the total Hamiltonian matrix is composed of three terms, as follows:

$$
\mathbf{H}\left(\mathbf{k}, \epsilon_{i j}\right)=\mathbf{H}_{\mathbf{k} \cdot \mathbf{p}}(\mathbf{k})+\mathbf{H}_{\mathrm{so}}+\mathbf{H}_{\epsilon}\left(\mathbf{k}, \epsilon_{i j}\right) .
$$

The first term is a doubly degenerate three band Hamiltonian matrix $\mathbf{H}_{\mathbf{k} \cdot \mathbf{p}}(\mathbf{k})$ calculated using the $\mathbf{k} \cdot \mathbf{p}$ method on the basis of $|x \uparrow\rangle,|y \uparrow\rangle,|z \uparrow\rangle,|x \downarrow\rangle,|y \downarrow\rangle$, and $|z \downarrow\rangle$. The three band structure parameters $L, M$, and $N$ adjust the Hamiltonian to fit experimental band structure data. ${ }^{20}$ The second term is a constant spin-orbit perturbation Hamiltonian matrix $\mathbf{H}_{\mathrm{so}}$ with a single parameter which is the spin-orbit splitting energy $\Delta_{\text {so }}$. The final term is a strain perturbation Hamiltonian matrix $\mathbf{H}_{\epsilon}\left(\mathbf{k}, \epsilon_{i j}\right)$, which is a function of both wavevector and strain tensor. The strain perturbation Hamiltonian has three deformation potential parameters $a, b$, and $d$ (Ref. 11), which are tuned to match experimental data. Since the present work
TABLE I. Parameter values for the $6 \times 6$ Hamiltonian (Ref. 21).

\begin{tabular}{ccccc}
\hline \hline $\begin{array}{c}L \\
\left(\mathrm{eV} \AA^{2}\right)\end{array}$ & $\begin{array}{c}M \\
(\mathrm{eV} \AA)\end{array}$ & $\begin{array}{c}N \\
(\mathrm{eV} \AA)\end{array}$ & $\begin{array}{c}d \\
(\mathrm{eV})\end{array}$ & $\begin{array}{c}\Delta_{\text {so }} \\
(\mathrm{eV})\end{array}$ \\
\hline-21.488 & -13.716 & -33.259 & -5.1 & 0.044 \\
\hline \hline
\end{tabular}

emphasizes the shear piezoresistance coefficient, only the shear deformation potential $d$ is used in this work. The parameter values used are listed in Table I. ${ }^{21}$ The total Hamiltonian is diagonalized at each $k$-point at a prescribed strain and six pair-wise identical eigenvalues are determined. These eigenvalues form the three valence bands. ${ }^{22}$

We base our TB calculation on the recent parametrization by Boykin et al. in Ref. 23. We found that earlier TB parameters developed by Jancu et al. $^{24}$ yield negative values for the piezocoefficient $\pi_{44}$ due to an erroneous $\pi / 2$ rotation of the band structure under pure shear strain. The effect of strain on these parameters is included both via the angular dependencies of the Slater-Koster decompositions, as well as the bond-length dependencies. ${ }^{25}$

\section{Numerical method}

The conductivity integral in the Boltzmann transport equation of Eq. (3) is calculated numerically using the discrete three-dimensional (3D) dispersion relation obtained using the band structure calculations outlined in the previous section. We assume full ionization so that $h=N_{A}$, where $h$ is the hole density. The density of holes may be calculated as

$$
h=\sum_{n} \frac{2}{(2 \pi)^{3}} \int f_{0}(\mathbf{k}, n) d \mathbf{k},
$$

where $f_{0}(\mathbf{k}, n)$ is the equilibrium distribution function for holes, and a factor of 2 for spin has been included. The sum extends over the three valence bands. By evaluating this integral for several values of the Fermi level $\xi_{F}$, we may determine the Fermi level at a given dopant level. To determine the strain dependence of the conductivity we calculate the 3D band structure of the strained material. However, to avoid numerical problems with the evaluation of the Fermi level integral, we do not calculate this for each strain. Instead, we have found it useful to determine the Fermi level of the unstrained structure, and then calculate the dependence of the Fermi level on strain along the desired direction as follows. Since the hole density is assumed to equal the constant $N_{A}$ independent of strain, we get

$$
\begin{aligned}
\frac{d h}{d \epsilon}= & \sum_{n} \int \frac{e^{\xi_{F}^{(0)}}-\xi_{n, \mathbf{k}}}{\left(e^{\xi_{F}^{(0)}}-\xi_{n, \mathbf{k}}+1\right)^{2}} \times \frac{d \xi_{n, \mathbf{k}}}{d \epsilon} d \mathbf{k} \\
& +\sum_{n} \int \frac{e^{\xi_{F}^{(0)}}-\xi_{n, \mathbf{k}}}{\left(e^{\xi_{F}^{(0)}}-\xi_{n, \mathbf{k}}+1\right)^{2}} \times \frac{d \xi_{F}}{d \epsilon} d \mathbf{k}=0,
\end{aligned}
$$

where $\xi_{F}$ and $\xi_{n, \mathbf{k}}$ are in units of $k T$. From this we calculate the change of Fermi level with strain, 
TABLE II. Values of $\pi_{44}$ in $p$-type silicon at an acceptor density of $N_{A}$ $=10^{14} \mathrm{~cm}^{-3}$ calculated using the TB model and the $6 \times 6$ model.

\begin{tabular}{ccc}
\hline \hline$T$ & $\mathrm{~TB} \pi_{44}$ & $6 \times 6 \pi_{44}$ \\
$(\mathrm{~K})$ & $\left(\times 10^{-11} \mathrm{~Pa}^{-1}\right)$ & $\left(\times 10^{-11} \mathrm{~Pa}^{-1}\right)$ \\
\hline 200 & 41 & 180 \\
300 & 31 & 122 \\
400 & 25 & 94 \\
\hline \hline
\end{tabular}

$$
\frac{d \xi_{F}}{d \epsilon}=\frac{\sum_{n} \int \frac{e^{\xi_{F}^{(0)}-\xi_{n, \mathbf{k}}}}{\left(e^{\left.\epsilon_{F}\right)}-\xi_{n, \mathbf{k}}+1\right)^{2}} \times \frac{d \xi_{n, \mathbf{k}}}{d \epsilon} d \mathbf{k}}{\sum_{n} \int \frac{e^{\xi_{F}^{(0)}-\xi_{n, \mathbf{k}}}}{\left(e^{\xi_{F}}-\xi_{n, \mathbf{k}}+1\right)^{2}} d \mathbf{k}},
$$

where $\xi_{F}^{(0)}$ is the Fermi level calculated at zero applied strain. We then calculate the Fermi level at a given strain as

$$
\xi_{F}(h, \epsilon)=\xi_{F}(h, \epsilon=0)+\frac{d \xi_{F}}{d \epsilon} \epsilon .
$$

Implementation of the model has been carried out in MATLAB. ${ }^{26}$ The calculated conductivities have all been converged with respect to the volume of $k$-space evaluated around the $\Gamma$ point as well as the $k$-point resolution. All results presented in this article have been calculated using equidistant $k$-point sampling along each principal axis. A total of $N=357911 k$-points have been sampled. The piezoresistance coefficients are extracted from a linear fit to calculated shear conductivities for four values of the applied shear strain $\epsilon_{x y} \in\{0,0.0005,0.001,0.0015,0.002\}$; in that strain range the relation between shear conductance and shear strain was almost perfectly linear.

\section{MODEL RESULTS}

Using the calculated conductivities with and without applied strain, the piezocoefficient $\pi_{44}$ is calculated from Eq. (2). We shall concentrate on $\pi_{44}$ due to the technological relevance compared to the other piezocoefficients $\pi_{11}$ and $\pi_{12}$; they could, however, be calculated in a manner similar to $\pi_{44}$, as seen from Eq. (1). In the calculation the shear compliance $S_{44}=1.256 \times 10^{-11} \mathrm{~Pa}^{-1}$ is used, ${ }^{27}$ while the relative temperature coefficient of $S_{44}\left(\sim 97 \times 10^{-6} \mathrm{~K}^{-1}\right)$ is ignored since the numerical value is much smaller than that of $\pi_{44}\left(\sim-3 \times 10^{-3} \mathrm{~K}^{-1}\right)$. The relative temperature coefficient of $S_{44}$ is estimated from the relative temperature coefficient of the coefficient of elasticity $C_{44}=1 / S_{44} \quad(\sim-97$ $\left.\times 10^{-6} \mathrm{~K}^{-1}\right)^{28}$

The values of $\pi_{44}$ at an acceptor density of $N_{A}=1.0$ $\times 10^{14} \mathrm{~cm}^{-3}$ calculated at temperatures $T=200,300$, and $400 \mathrm{~K}$ using the TB model and the $6 \times 6$ model are listed in Table II. Calculations of $\pi_{44}$ showing the detailed dependency on acceptor dopant density and temperature are shown graphically in Fig. 1 when using the $6 \times 6$ model. The two different models result in similar observable trends for the temperature as well as the dopant density dependency. In the temperature range from $T=200$ to $450 \mathrm{~K}$ the $\pi_{44}$ value extracted from the TB model varies from $41 \times 10^{-11}$ to 22 $\times 10^{-11} \mathrm{~Pa}^{-1}$ at the dopant density $N_{A}=1.0 \times 10^{14} \mathrm{~cm}^{-3}$.

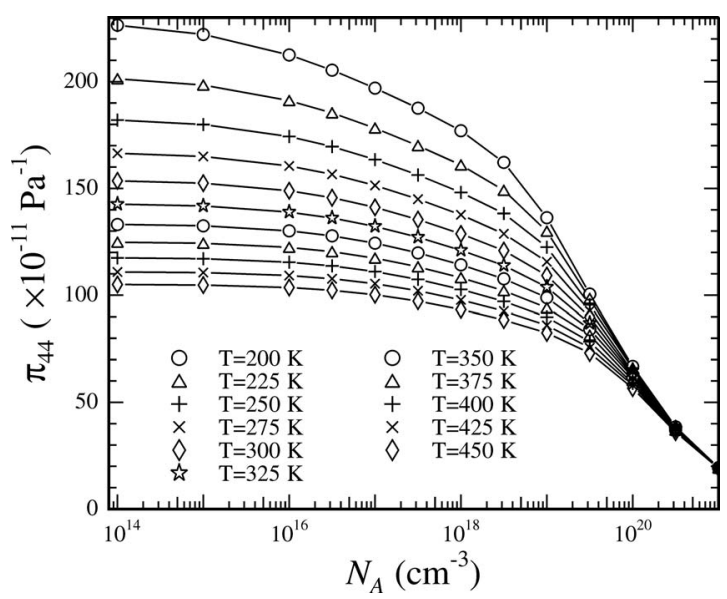

FIG. 1. The piezocoefficient $\pi_{44}$ as a function of carrier density and temperature calculated using the $6 \times 6$ model. The calculated values are in good agreement with the experimental data listed in Table III.

Compared to published experimental data listed in Table III, these values are approximately a factor of 4 too small. Thus, we conclude that the parameters used in the TB model are not yet fully optimized for piezoresistance calculations. In contrast to this large discrepancy, the $6 \times 6$ model results in absolute $\pi_{44}$ values essentially in agreement with experiments.

In Fig. 2 the calculated temperature dependency, for both the $6 \times 6$ and TB models, is illustrated by plotting the $\pi_{44}$ values normalized with the values calculated at $T=300 \mathrm{~K}$ for two different doping levels, $N_{A}=1.0 \times 10^{14}$ and 1.0 $\times 10^{19} \mathrm{~cm}^{-3}$. The normalized piezocoefficient values are plotted as a function of $1 / T$. At low dopant density the piezocoefficients calculated from the $6 \times 6$ model closely follow the expected $1 / T$ dependency, while the temperature dependency extracted from the TB model is somewhat smaller (by approximately 10\%). At the higher dopant density the calculated temperature dependencies from the two models are similar and less than the $1 / T$ dependency. The approximate $1 / T$ dependencies arise from piezocoefficients approximately inversely proportional to the kinetic energy of the carriers, while the similar dependency observed in $n$-type silicon is due to repopulation effects.

TABLE III. Experimental values of the piezocoefficient $\pi_{44}$ in $p$-type silicon at $T=300 \mathrm{~K}$. The last column lists standard deviations of the piezocoefficient measurements when available.

\begin{tabular}{cccc}
\hline \hline Ref. & $\begin{array}{c}\text { Dopant density } \\
\left(\times 10^{18} \mathrm{~cm}^{-3}\right)\end{array}$ & $\begin{array}{c}\pi_{44} \\
\left(\times 10^{-11} \mathrm{~Pa}^{-1}\right)\end{array}$ & $\begin{array}{c}\text { Deviation } \\
(\%)\end{array}$ \\
\hline 1 & 0.002 & 138.1 & \\
29 & 0.02 & 93.1 & 7.5 \\
30 & 0.03 & 113.5 & 6 \\
31 & 0.8 & 105 & $8-12$ \\
32 & 1.5 & 87 & 6.5 \\
5 & 3 & 111 & \\
31 & 8.2 & 95 & \\
5 & 9 & 98 & \\
5 & 50 & 78 & \\
5 & 300 & 60 & \\
5 & 500 & 48 & \\
5 & 2000 & 35 & \\
\hline
\end{tabular}




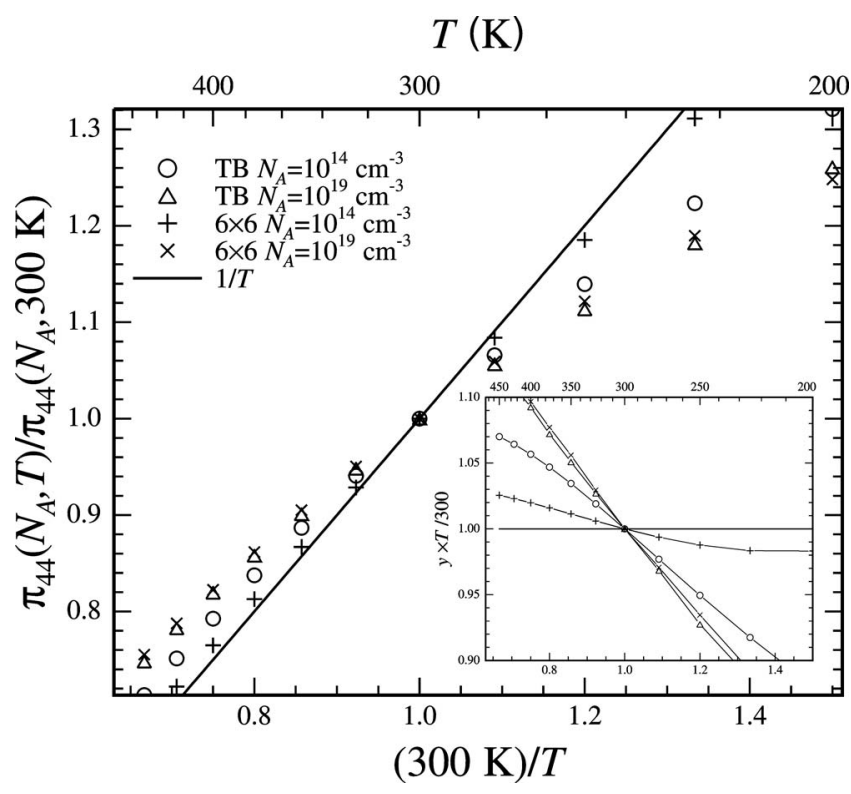

FIG. 2. Temperature dependency of the normalized piezocoefficient $\pi_{44}(T) / \pi_{44}\left(T_{0}=300 \mathrm{~K}\right)$ at the dopant densities $N_{A}=1.0 \times 10^{14}$ and 1.0 $\times 10^{19} \mathrm{~cm}^{-3}$ calculated using the TB model and the $6 \times 6$ model. The solid line shows a perfect $1 / T$ dependency. In the inset the normalized piezocoefficients are multiplied by the factor $T / 300 \mathrm{~K}$ to reveal the deviation from the expected $1 / T$ dependency. The $6 \times 6$ model calculations at low acceptor density $\left(N_{A}=1.0 \times 10^{14} \mathrm{~cm}^{-3}\right)$ deviates only a few percent from the $1 / T$ dependency.

\section{EXPERIMENTAL}

We have designed and fabricated piezoresistance test chips in order to compare the calculated piezocoefficients from the two models with experimental data. A micrograph of the chip is shown in Fig. 3. In the region near the center of the chip, six resistors, oriented along different crystal directions, are defined. The chips are fabricated using conventional microfabrication techniques. The test chips have been fabricated in two different processes. One process includes the use of a silicon on insulator (SOI) substrate with a (001) device layer; the SOI starting material is very useful since it facilitates uniform doping of the piezoresistors. The piezoresistors are defined using reactive ion etching (RIE), and boron doped using ion implantation followed by a thermal oxidation and anneal to ensure activation and uniform doping of the device layer. The uniformly doped piezoresistors have doping concentrations of $N_{A}=1.5 \times 10^{17}, 2.0 \times 10^{18}$, and 2.2

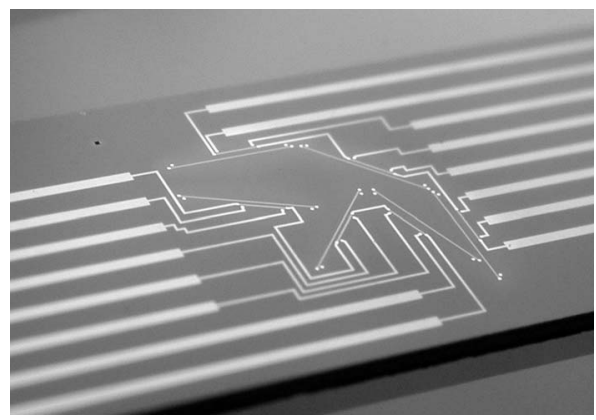

FIG. 3. Close-up micrograph of the fabricated piezoresistance test chip. Six piezoresistors are directed along different directions and located in the center region of the chip. The chip is $4 \mathrm{~cm}$ long and $5.3 \mathrm{~mm}$ wide.

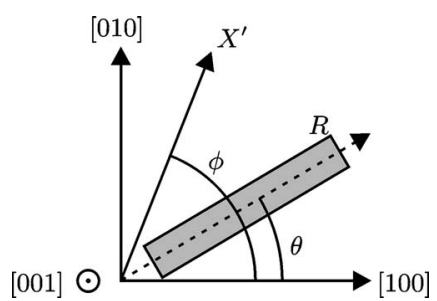

FIG. 4. Schematic showing the orientation of the piezoresistor and the applied uniaxial stress $X^{\prime}$. The long axis of the resistor is oriented at the angle $\theta$, while the uniaxial stress is applied at the angle $\phi$ to the [100] direction on a (001) substrate.

$\times 10^{19} \mathrm{~cm}^{-3}$. In the other process boron ion implantation on the surface of a (001) silicon substrate followed by RIE, thermal oxidation, and anneal gives piezoresistors a Gaussian doping profile. The peak boron concentrations in the Gaussian doping profile resistors are $N_{A}=9.1 \times 10^{17}, 9.4$ $\times 10^{18}$, and $4.6 \times 10^{19} \mathrm{~cm}^{-3}$. For both processes contact windows are etched using buffered $\mathrm{HF}$ and interconnects to the resistors are defined in an e-beam evaporated double layer of $\mathrm{Ti} / \mathrm{Al}$. The piezoresistance characterization is done in an automated four point bending fixture. This fixture applies a uniaxial, uniform stress to the resistors in the center region of the chip $^{33}$ in steps of approximately 5 to a maximum value of $70 \mathrm{MPa}$, which corresponds to a strain of $\epsilon_{x x} \approx 0.0004$.

In the experimental approach we use Eq. (1) where the change in resistivity depends on the applied stress $X$. The relative change in resistivity is equal to the relative change in the resistance of a piezoresistor. Now consider the resistor $R$ on a (001) substrate as shown in Fig. 4. By applying the uniaxial, uniform stress $X^{\prime}$ to the sample, the relative resistance change is ${ }^{34}$

$$
\begin{aligned}
\frac{\Delta R}{R_{0}}(\phi, \theta)= & \frac{X^{\prime}}{2}\left[\pi_{11}+\pi_{12}+\cos (2 \phi) \cos (2 \theta)\left(\pi_{11}-\pi_{12}\right)\right. \\
& \left.+\sin (2 \theta) \sin (2 \phi) \pi_{44}\right]
\end{aligned}
$$

where $\theta$ is the angle of the resistor orientation with respect to the [100] direction and $\phi$ is the angle at which the stress $X^{\prime}$ is directed with respect to the [100] direction. The piezocoefficient $\pi_{44}$ can then be found by measuring the resistance on two resistors directed according to the equation

$$
\pi_{44}=\frac{\Delta R}{R_{0}}\left(\frac{\pi}{4}, \frac{\pi}{4}\right) \frac{1}{X^{\prime}}-\frac{\Delta R}{R_{0}}\left(\frac{\pi}{4}, \frac{3 \pi}{4}\right) \frac{1}{X^{\prime}}=\pi_{l}-\pi_{t},
$$

where $\pi_{l}$ and $\pi_{t}$ are the longitudinal and transversal piezocoefficients, respectively.

\section{DISCUSSION}

Using the piezoresistance test chips in the four point bending setup, we have experimentally determined the shear piezocoefficient $\pi_{44}$ in silicon with the acceptor dopant densities given in Sec. IV at three different temperatures $T$ $=300,325$, and $350 \mathrm{~K}$. The experimental data are plotted in Fig. 5, where experimental data from Tufte and Stelzer ${ }^{5}$ are added to extend the experimental range of dopant densities and temperatures. Our measurements are seen to agree well with the data from literature in the range where the param- 


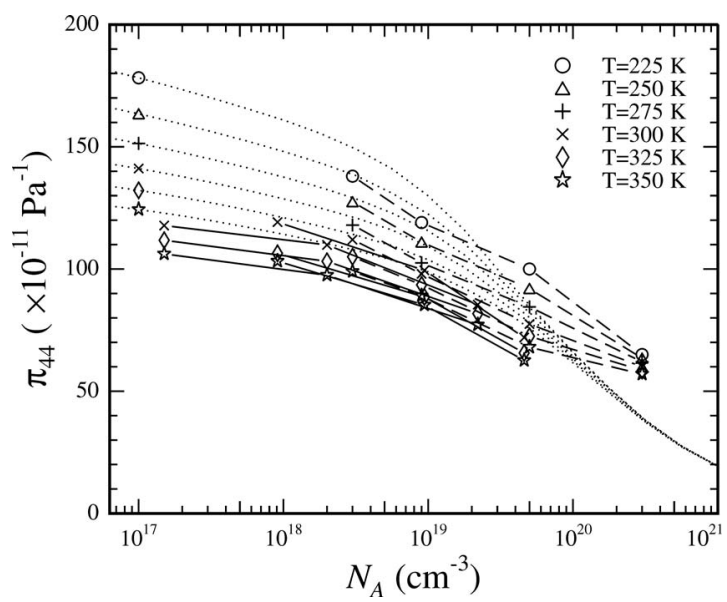

FIG. 5. Experimental and calculated values of the piezocoefficient $\pi_{44}$ as a function of doping level with temperature as parameter. The dashed lines are experimental data from Tufte and Stelzer (Ref. 5) and the solid lines are experimental data obtained from the piezoresistors described in Sec. IV. Notice that the full lines connect the actual experimental points and are thus just guides to the eye. The dotted lines are model calculations using the 6 $\times 6$ model.

eters are comparable. In Fig. 5 the experimental data are compared to shear piezocoefficients $\pi_{44}$ calculated using the $6 \times 6$ model. The absolute magnitude of the calculated piezocoefficients are in quite good agreement with the measured values, and any small discrepancy at a given doping level and temperature could be attributed to the value of the shear deformation potential $d$ used, since $\pi_{44} \propto d$.

To focus attention on the temperature and doping level dependency, we plot in Fig. 6 the data of Fig. 5 normalized to $\pi_{44}$ at $T=300 \mathrm{~K}$ and $N_{A}=3.0 \times 10^{18} \mathrm{~cm}^{-3}$. Excellent agreement between experiments and model calculations is seen at dopant densities below $1 \times 10^{19} \mathrm{~cm}^{-3}$. At larger dopant densities the model calculations show a significantly stronger dopant density dependency than experimentally observed. This discrepancy could be due to several reasons related to shortcomings in the model used. First, in the model

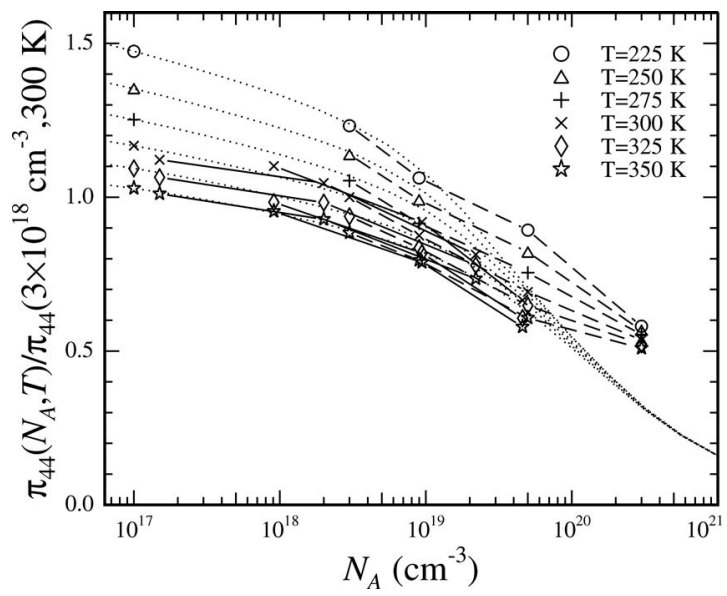

FIG. 6. Experimental and calculated values of the normalized piezocoefficient $\pi_{44}$ as a function of doping level with temperature as parameter. The piezocoefficients are normalized to $\pi_{44}$ at $T=300 \mathrm{~K}$ and $N_{A}=3.0$ $\times 10^{18} \mathrm{~cm}^{-3}$. The dashed lines are experimental data from Tufte and Stelzer (Ref. 5) and the solid lines are experimental data obtained from the piezoresistors described in Sec. IV. Notice that the full lines connect the actual experimental points and are thus just guides to the eye. The dotted lines are model calculations using the $6 \times 6$ model.

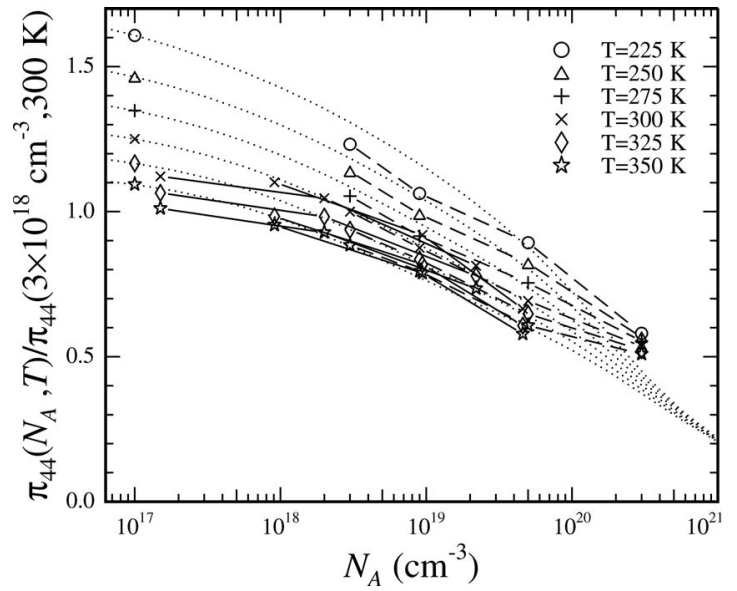

FIG. 7. Experimental and calculated values of the normalized piezocoefficient $\pi_{44}$ as a function of doping level with temperature as parameter. The piezocoefficients are normalized to $\pi_{44}$ at $T=300 \mathrm{~K}$ and $N_{A}=3.0$ $\times 10^{18} \mathrm{~cm}^{-3}$. The dashed lines are experimental data from Tufte and Stelzer (Ref. 5) and the solid lines are experimental data obtained from the piezoresistors described in Sec. IV. Notice that the full lines connect the actual experimental points and are thus just guides to the eye. The dotted lines are model calculations using the $6 \times 6$ model with the scattering rates multiplied by the availability factor $\left(1-f_{0}\right)$.

of the scattering rates we have ignored the effect of availability of the final states; this is vital at very high doping levels. Second, at very high doping the band structure is modified by band tailing. ${ }^{35}$ As a result, the actual Fermi level at high doping will be closer to the band edge than expected from simple theory. This is easily understood if we write the total hole concentration $h=h_{\mathrm{bt}}+h_{b}$, where $h_{\mathrm{bt}}$ is the hole concentration in the band tail while $h_{b}$ is the hole concentration in the remaining unperturbed bands. This change in doping level dependency of the Fermi level will affect the magnitude of the piezocoefficient as well as the temperature dependency, since relatively simple considerations predict a piezocoefficient $\pi_{44} \propto d /\left\langle E_{\text {kin }}\right\rangle$, where $\left\langle E_{\text {kin }}\right\rangle$ is the average kinetic energy of the carriers contributing to the conductivity. At low doping levels $\left\langle E_{\mathrm{kin}}\right\rangle \propto k_{B} T$ while at very high doping levels only carriers with a kinetic energy close to the Fermi level contribute to the conductivity and $\left\langle E_{\text {kin }}\right\rangle$ equals the energy difference between the Fermi level and the band edge. Thus the temperature dependency of the piezocoefficient vanishes at high doping levels, as is also seen in the calculations.

To illustrate the effect of availability of final states we adopt a crude model, where the scattering rate Eq. (5) is multiplied by the availability factor $\left(1-f_{0}\right)$, and calculate the resulting piezocoefficients using the $6 \times 6$ model. The result of the calculation is shown in Fig. 7 where piezocoefficients normalized to $\pi_{44}$ at $T=300 \mathrm{~K}$ and doping level $N_{A}=3.0$ $\times 10^{18} \mathrm{~cm}^{-3}$ are shown along with the experimental values. The agreement between model calculations and experiments is seen to improve; at dopant densities below 1 $\times 10^{20} \mathrm{~cm}^{-3}$ the agreement is very good. The remaining disagreement is probably due to the band-tailing effect discussed above. Unfortunately, a simple yet satisfactory model for this effect is not available; therefore we have not been able to do modeling experiments to verify the effect on the piezocoefficients. 


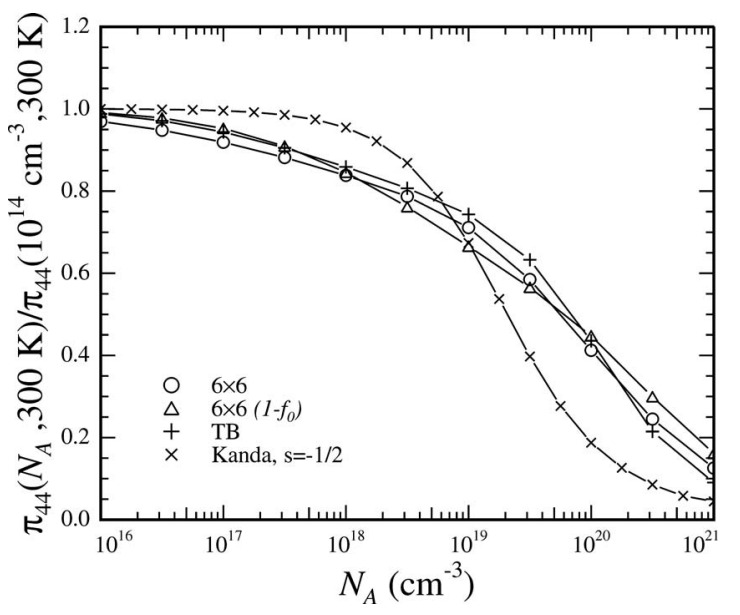

FIG. 8. Calculated piezocoefficients $\pi_{44}$ at $T=300 \mathrm{~K}$ normalized to $\pi_{44}$ at low dopant density $N_{A}=1.0 \times 10^{14} \mathrm{~cm}^{-3}$ as a function of acceptor dopant density. Calculations using both the TB and the $6 \times 6$ models are shown. For the $6 \times 6$ model a calculation using the scattering rate multiplied by the availability factor $\left(1-f_{0}\right)$ is added. Finally, the correction factor due to Kanda (Ref. 6) using $s=-1 / 2$ is included.

To illustrate the effect of the band structure model and the scattering rate model we plot the calculated normalized piezocoefficients at $T=300 \mathrm{~K}$ resulting from the $6 \times 6$ and TB models in Fig. 8, where a calculation for the $6 \times 6$ model with the scattering rate multiplied by the availability factor $\left(1-f_{0}\right)$ is also shown. The piezocoefficients are normalized with $\pi_{44}$ at very low dopant density $N_{A}=1.0 \times 10^{14} \mathrm{~cm}^{-3}$. Finally, for comparison, the popular correction factor due to Kanda ${ }^{6}$ with $s=-1 / 2$ is added to the graph. The dopant density dependency of all our model calculations differ significantly from Kanda's correction factor. The effect of increased doping density is much more gradual in our calculations. The dopant density dependency predicted from the two band structure models differ only slightly.

The effect of the scattering rate model on the calculated piezocoefficients from the $6 \times 6$ model is shown in Fig. 9, where the calculated piezocoefficients at $T=300 \mathrm{~K}$ normal-

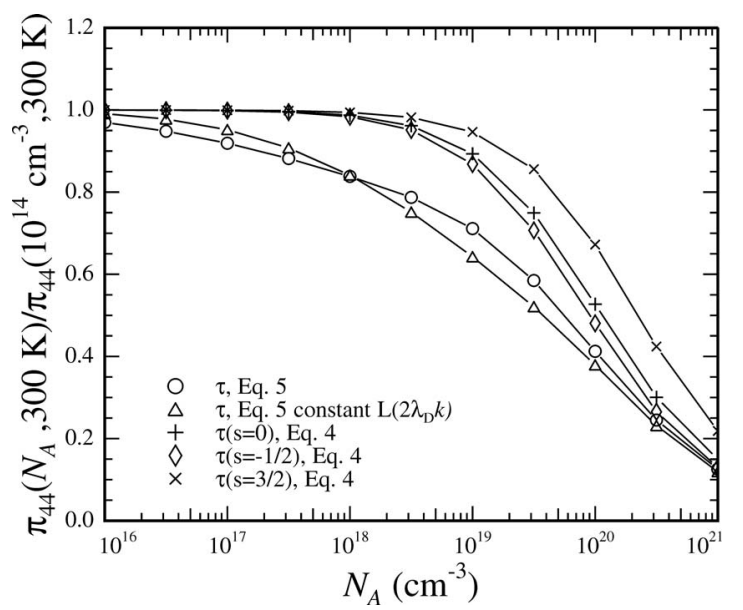

FIG. 9. Piezocoefficients $\pi_{44}$ at $T=300 \mathrm{~K}$ normalized to $\pi_{44}$ at low dopant density $N_{A}=1.0 \times 10^{14} \mathrm{~cm}^{-3}$ calculated from the $6 \times 6$ model as a function of acceptor dopant density. Five different models for the scattering rates are used. The results from using the full scattering rate model Eq. (5) and the same model with a slightly simplified impurity scattering model replacing $L\left(2 \lambda_{D} k\right)$ by a constant are shown together with calculations using the power law model Eq. (4) using $s=0,-1 / 2$, and $3 / 2$.
TABLE IV. Fitting parameters used in Eq. (20).

\begin{tabular}{cc}
\hline \hline Parameter & Value \\
\hline$N_{b}$ & $6 \times 10^{19} \mathrm{~cm}^{-3}$ \\
$N_{c}$ & $7 \times 10^{20} \mathrm{~cm}^{-3}$ \\
$\vartheta$ & 0.9 \\
$\alpha$ & 0.43 \\
$\beta$ & 0.1 \\
$\gamma$ & 1.6 \\
$\eta$ & 3 \\
\hline \hline
\end{tabular}

ized to $\pi_{44}$ at very low dopant density $N_{A}=1.0 \times 10^{14} \mathrm{~cm}^{-3}$ are shown for five different models of the scattering rate. The results from using the full scattering rate model Eq. (5) and the same model with a slightly simplified impurity scattering model replacing $L\left(2 \lambda_{D} k\right)$ by a constant are shown together with calculations using the power law model Eq. (4) using $s=0,-1 / 2$, and $3 / 2$. Obviously, if all relevant scattering mechanisms are included, a much more gradual reduction in the piezocoefficient with increased doping level is seen as compared to the steep reduction obtained with the power law models. Since the gradual decrease is also seen experimentally (Fig. 5), we conclude that it is essential to use the detailed scattering rate model. The effect of using a less accurate impurity scattering model is quite small though.

\section{A. Piezocoefficient fit}

In order to produce a more satisfactory correction factor compared to that of Kanda, ${ }^{6}$ we fit the normalized piezocoefficient $\pi_{44}$ as a function of temperature and acceptor density to the results from the $6 \times 6$ model calculations with the scattering rate multiplied by the availability factor $\left(1-f_{0}\right)$. We fit the normalized piezocoefficient to the function

$$
P\left(N_{A}, \Theta\right)=\Theta^{-\vartheta}\left[1+\left(\frac{N_{A}}{N_{b}}\right)^{\alpha} \Theta^{-\beta}+\left(\frac{N_{A}}{N_{c}}\right)^{\gamma} \Theta^{-\eta}\right]^{-1},
$$

where $P\left(N_{A}, \Theta\right)$ is the piezocoefficient normalized to the lowest acceptor density value at $T_{0}=300 \mathrm{~K}, \Theta=T / T_{0}, N_{b}$ and $N_{c}$ are fitting parameters, and $\vartheta, \alpha, \beta, \gamma$, and $\eta$ are power coefficients.

The fitting parameters are found by a two-dimensional fit to the surface defined by $N_{A}$ and $\Theta$. The resulting parameters are listed in Table IV. The first term in the denominator dominates at low acceptor densities while at high acceptor densities both terms are important. The fit is shown in Fig. 10 together with normalized piezocoefficient values calculated using the $6 \times 6$ model. The simple fit agrees well with the $6 \times 6$ model calculations and can therefore be used to predict the $\pi_{44}$ dependence on acceptor density and temperature at acceptor densities below $1 \times 10^{20} \mathrm{~cm}^{-3}$. Thus, the fitting function is a simple tool provided to experimentalist and industrial MEMS developers within piezoresistive device research and applications.

\section{CONCLUSION}

We have numerically calculated the shear piezocoefficient $\pi_{44}$ in $p$-type silicon using a $6 \times 6 \mathbf{k} \cdot \mathbf{p}$ Hamiltonian 


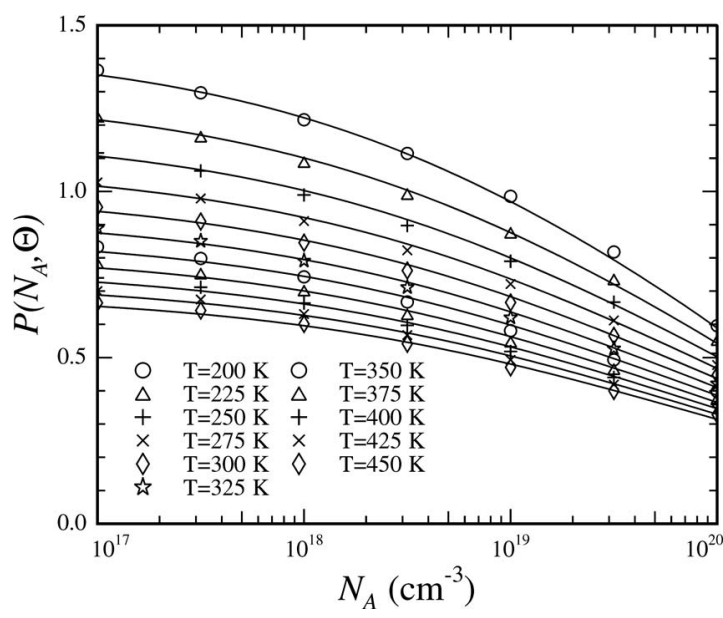

FIG. 10. Fitted correction factor for $\pi_{44}$ as a function of carrier density and temperature. The fit (solid) is described by Eq. (20) and the fitting parameters are listed in Table IV. The symbols are $6 \times 6$ model calculations using the availability corrected scattering rates.

band structure calculation combined with detailed analytical models for the scattering rates. These results have been compared to results obtained with a recent TB model as well as to experimental data. We have measured the shear piezocoefficient $\pi_{44}$ in $p$-type silicon at several dopant densities and temperatures, using dedicated test chips and a four point bending fixture measurement setup. Band structures calculated from the $6 \times 6 \mathbf{k} \cdot \mathbf{p}$ Hamiltonian predict piezocoefficients in quite good agreement with experiments at acceptor dopant densities below $N_{A}=1 \times 10^{20} \mathrm{~cm}^{-3}$. We have found that it is important to include all relevant scattering mechanisms, acoustic phonon, nonpolar optical phonon, and ionized impurity scattering in order to get decent agreement between model calculations and experiments. This is particularly important in order to reproduce the gradual decrease in piezoresistance coefficient with increased dopant density.

Our comparison highlights the importance of an accurate description of the strain dependence of TB parameters. The parameters developed by Boykin et al. result in a strained band structure that underestimates the piezocoefficients by a factor of 4 compared to the experiments and the $6 \times 6 \mathbf{k} \cdot \mathbf{p}$ results. It follows that more work is needed in order to optimize TB parameters for piezoresistance calculations.

The calculations result in a piezoresistance coefficient variation with temperature quite close to $1 / T$ dependency at low dopant density. This dependency is caused by the average kinetic energy of the carriers contributing to the conductivity. In $p$-type silicon repopulation effects are insignificant, whereas these are the reason for the similar $1 / T$ dependency seen in $n$-type silicon. ${ }^{7}$

Finally, since the piezocoefficient model calculations are in quite good agreement with experiments, we have developed a simple analytical fit to the calculated piezocoefficients; this fit could prove to be a useful tool in device modeling and optimization.

\section{ACKNOWLEDGMENTS}

We thank Dr. Chris Bowen (Texas Instrument) for useful comments on the TB parametrizations. Center for Individual Nanoparticle Functionality (CINF) is sponsored by The Danish National Research Foundation.
${ }^{1}$ C. S. Smith, Phys. Rev. 94, 42 (1954).

${ }^{2}$ O. N. Tufte, P. W. Chapman, and D. Long, J. Appl. Phys. 33, 3322 (1962).

${ }^{3}$ J. Richter, O. Hansen, A. Nylandsted Larsen, J. Lundsgaard Hansen, G. F. Eriksen, and E. V. Thomsen, Sens. Actuators, A 123-124, 388 (2005).

${ }^{4}$ R. He and P. Yang, Nanotechnology 1, 42 (2006).

${ }^{5}$ O. N. Tufte and E. L. Stelzer, J. Appl. Phys. 34, 313 (1963).

${ }^{6}$ Y. Kanda, IEEE Trans. Electron Devices 29, 64 (1982).

${ }^{7}$ C. Herring and E. Vogt, Phys. Rev. 101, 944 (1956).

${ }^{8}$ E. N. Adams, Phys. Rev. 96, 803 (1954).

${ }^{9}$ J. A. Harley and T. W. Kenny, J. Microelectromech. Syst. 9, 226 (2000).

${ }^{10}$ S. I. Kozlovskiy, V. V. Nedostup, and I. I. Boiko, Sens. Actuators, A 133, 72 (2007).

${ }^{11}$ G. E. Pikus and G. L. Bir, Symmetry and Strain-Induced Effects in Semiconductors (Wiley, New York, 1974).

${ }^{12}$ T. Toriyama and S. Sugiyama, J. Microelectromech. Syst. 11, 598 (2002).

${ }^{13}$ K. Suzuki, H. Hasegawa, and Y. Kanda, Jpn. J. Appl. Phys., Part 2 23, L871 (1984).

${ }^{14}$ P. Kleimann, B. Semmache, M. Le Berre, and D. Barbier, Phys. Rev. B 57, 8966 (1998).

${ }^{15}$ Y. Ohmura, J. Phys. Soc. Jpn. 61, 217 (1992).

${ }^{16}$ J. F. Nye, Physical Properties of Crystals, 1st ed. (Oxford University Press, New York, 1957).

${ }^{17}$ B. M. Askerov, Electron Transport Phenomena in Semiconductors (World Scientific, Singapore, 1994).

${ }^{18}$ J. Singh, Physics of Semiconductors and Their Heterostructures (McGraw-Hill, New York, 1993).

${ }^{19}$ J. M. Hinckley and J. Singh, Phys. Rev. B 41, 2912 (1990).

${ }^{20}$ G. Dresselhaus, A. F. Kip, and C. Kittel, Phys. Rev. 98, 368 (1955).

${ }^{21}$ P. Y. Yu and M. Cardona, Fundamentals of Semiconductors, Physics and Material Properties (Springer-Verlag, Berlin, 2001).

${ }^{22}$ J. C. Slater and G. F. Koster, Phys. Rev. 94, 1498 (1954).

${ }^{23}$ T. B. Boykin, G. Klimeck, and F. Oyafuso, Phys. Rev. B 69, 115201 (2004).

${ }^{24}$ J. M. Jancu, R. Scholz, F. Beltram, and F. Bassani, Phys. Rev. B 57, 6493 (1998).

${ }^{25}$ The TB model uses a basis set consisting of ten orthogonal valence orbitals $\left(s p^{3} d^{5} s^{*}\right)$. The bond-length dependencies of the two-center integrals are fitted to generalizations of Harrison's free-electron distance law (Refs. 36 and 37). On-site parameters are shifted due to the displacements of nearest neighbors (Ref. 38). The spin-orbit is taken into account in the model by adding intra-atomic matrix elements using Chadi's approximation (Ref. 39).

${ }^{26}$ MATLAB version 7.0.1.

${ }^{27}$ S. K. Clark and K. D. Wise, IEEE Trans. Electron Devices 26, 1887 (1979).

${ }^{28}$ H. Metzger and F. R. Kessler, Z. Naturforsch. A 25, 904 (1970).

${ }^{29}$ R. E. Beaty, R. C. Jaeger, J. C. Suhling, R. W. Johnson, and R. D. Butler, IEEE Trans. Compon., Hybrids, Manuf. Technol. 15, 904 (1992).

${ }^{30}$ R. C. Jaeger, J. C. Suhling, and A. A. Anderson, Proceedings of the 44th IEEE Electronic Components and Technology Conference, p. 741-749 (1994).

${ }^{31}$ E. Lund and T. Finstad, InterPACK '99: Pacific RIM/ASME International Intersociety Electronics Photonic Packaging Conference, Advances in Electronic Packaging, p. 215-218 (1999).

${ }^{32}$ R. C. Jaeger, J. C. Suhling, M. T. Carey, and R. W. Johnson, IEEE Trans. Compon., Hybrids, Manuf. Technol. 16, 925 (1993).

${ }^{33}$ J. Richter, M. B. Arnoldus, O. Hansen, and E. V. Thomsen, Rev. Sci. Instrum. 79, 044703 (2008).

${ }^{34}$ D. A. Bittle, J. C. Suhling, R. E. Beaty, R. C. Jaeger, and R. W. Johnson, J. Electron. Packag. 113(3), 203 (1991).

${ }^{35}$ S. Wang, Fundamentals of Semiconductor Theory and Device Physics (Prentice-Hall, Englewood Cliffs, NJ, 1989).

${ }^{36}$ W. A. Harrison, Electronic Structure and Properties of Solids (Dover, New York, 1989).

${ }^{37}$ T. B. Boykin, N. Kharche, and G. Klimeck, Phys. Rev. B 76, 035310 (2007).

${ }^{38}$ T. B. Boykin, G. Klimeck, R. C. Bowen, and F. Oyafuso, Phys. Rev. B 66, 125207 (2002)

${ }^{39}$ D. J. Chadi, Phys. Rev. B 16, 790 (1977). 